\title{
Chloride Channel Binding
}

National Cancer Institute

\section{Source}

National Cancer Institute. Chloride Channel Binding. NCI Thesaurus. Code C40484.

A process that involves the binding of calcium or an extracellular ligand to a calcium channel. These interactions are involved in restoring the electrochemical potential of the resting membrane. 